\title{
Notion d'intérêt public et avenir de la conservation de la nature en République Démocratique du Congo
}

\author{
Ursil Lelo Di Makungu / Daddy Bogole Bolimia / Juvénal Madigo Ntekenge / \\ Blaise Iyamba Valentin / Richard Mandandi Akemane / Martin Amisa Zogi / Nady- \\ ne-Clémence Chalachala / Ibrahim Tshimpanga / Didier Okoto Lofongola / Au- \\ gustin Bedidjo Ular*
}

* Ursil Lelo DI MAKUNGU est Docteur en Droit Public de la Faculté de Droit de KU Leuven en Belgique et Docteur en Droit Economique et Social de la Faculté de Droit de l'Université de Kisangani, il est Professeur à la Faculté de Droit de l'Université de Kisangani et Vice-Doyen des recherches à la Faculté de Droit au sein de la même Université. Avocat au Barreau de Kisangani et Directeur du Centre de Recherche Interdisciplinaire de Droit, Gouvernance Territoriale et Développement Durable. Courriel : lelodims@gmail.com/lelo.dimakungu@unikis.ac.cd;

Daddy BOGOLE BOLIMIA, Economiste, Master en Gestion de la Biodiversité et Aménagement Forestier Durable. Chercheur Sénior et Directeur Adjoint du Centre de Recherche Interdisciplinaire de Droit, Gouvernance Territoriale et développement Durable. Courriel : dbogole@gmail.com;

Juvénal MADIGO NTEKENGE, Avocat au Barreau de Kisangani et Chercheur Sénior au Centre de Recherche Interdisciplinaire de Droit, Gouvernance Territoriale et développement Durable. Courriel : juvnalmadigo@gmail.com;

Blaise IYAMBA Valentin, Avocat au Barreau de Kisangani et Chercheur Sénior au Centre de Recherche Interdisciplinaire de Droit, Gouvernance Territoriale et développement Durable. Assistant à la Faculté de Droit de l'Université Kisangani. Depuis le 06 septembre 2019, il est master en droits de l'homme des Universités Saint Louis de Bruxelles, Catholique de Louvain et Namur en Belgique. Courriel : iyambablaise@gmail.com;

Richard MANDANDI AKEMANE, Avocat au Barreau de Kisangani et Chercheur Sénior au Centre de Recherche Interdisciplinaire de Droit, Gouvernance Territoriale et développement Durable. Courriel : richomandandi@gmail.com;

Martin AMISA ZOGI, Avocat au Barreau de Kisangani et Chercheur Sénior au Centre de Recherche Interdisciplinaire de Droit, Gouvernance Territoriale et Développement Durable. Courriel : amzomamisa@gmail.com;

Nadyne-Clémence CHALACHALA, Avocate au Barreau de Kisangani, doctorante à la Faculté de Droit de l'Université de Kisangani et Chercheuse Sénior au Centre de Recherche Interdisciplinaire de Droit, Gouvernance Territoriale et Développement Durable. Courriel : nadyaclemence2009@gm ail.com;

Ibrahim TSHIMPANGA, Avocat au Barreau de Kisangani, Assistant à la Faculté de Droit de l'Université de l'Uélé à Isiro et Chercheur Sénior au Centre de Recherche Interdisciplinaire de Droit, Gouvernance Territoriale et Développement Durable. Courriel : ibrahimtshimpanga@gmail.com;

Didier OKOTO LOFONGOLA, Juriste et Chercheur Sénior au Centre de Recherche Interdisciplinaire de Droit, Gouvernance Territoriale et Développement Durable. Courriel : okotodieudonne@g mail.com;

Augustin BEDIDJO ULAR, Avocat au Barreau de Kisangani, chercheur Senior au Centre de Recherche Interdisciplinaire de Droit, Gouvernance Territoriale et Développement Durable. Il est également Assistant à la Faculté de Droit de l’Université de Kisangani. Courriel : augustinular@ymail.c om 


\section{Résumé}

La gouvernance durable des aires protégées est généralement motivée au nom de l'intérêt général en dépit de l'imprécision juridique qui caractérise cette notion. Afin d'assurer cette gouvernance durable pour la conservation des aires protégées, la République Démocratique du Congo (RDC), a besoin non seulement des moyens matériels, financiers et humains, mais surtout des politiques publiques clairement définies et orientées vers la conservation durable de ses ressources matérialisées dans un instrument juridique sectoriel. Par ailleurs, la loi $\mathrm{n}^{\circ} 14 / 003 \mathrm{du} 11$ février 2014 relative à la conservation de la nature apparait comme le véritable instrument efficace et adapté de la concrétisation et de la mise en œuvre de la politique publique en matière de gestion durable des aires protégées. Cependant l'usage arbitraire de cette notion d'intérêt général sans contenu juridique clair risque de paraître plutôt comme une géomancie juridique et administrative pour porter atteinte à la conservation de la nature, à la suite de l'absence de l'échelle de valeur pour mieux cerner d'une part, les mesures de conservation et de l'autre, les dérogations à ces mesures. Suite à cette difficulté d'évaluation des valeurs liées à la conservation et aux restrictions y afférentes, le législateur entretient un flou offrant ainsi un pouvoir exorbitant au pouvoir public à l'Etat et aux entités territoriales au nom de l'intérêt de pouvoir. Certains acteurs de la politique publique s'accordent des pouvoirs arbitraires au nom de cet intérêt afin de substituer une zone de conservation à une autre activité contraire aux objectifs et principes de conservation durable des aires protégées. Cette étude entend aborder la notion d'intérêt public qui du reste, sujette à des diverses appréhensions et conception tant par le pouvoir public que par l'opinion publique, suite à sa vitalité dans une perspective spatio-temporelle. Les indicateurs de l'analyse de conciliation du concept d'intérêt public au contexte du droit congolais de la conservation de la nature révèlé plutôt être une pure supercherie intellectuelle instaurée par le législateur et dont le pouvoir public s'en sert pour enfreindre les mesures de conservation de la nature. C'est autour de toute ces questions que la présente étude à bien voulu répondre aux préoccupations de la compréhension de la notion d'intérêt public, son contenu, ses critères et fonctions, son utilité, le lien qu'il entretien avec le droit de la conservation et son avenir dans le domaine de la conservation de la nature en RDC.

\section{Introduction}

Les ressources naturelles et biologiques dont regorge la RDC sont immenses et diversifiées et qui, au regard de leur importance tant sur le plan national qu'international mérite une attention particulière du pouvoir public, à travers les politiques publiques de la conservation de la nature. Ces orientations politiques sont prises sur le plan mondial par les personnes publiques de droit international, de même sur le plan national, provincial et local par les autorités compétentes, dans le seul but de permettre un grand nombre de populations mon-

Le premier auteur remercie le CIFOR et l'Université de Kisangani dans le cadre du projet FORETS pour le soutien dans la rédaction du présent article. 
diales de répondre à leur besoin économique, social et environnemental, tout en garantissant ceux des générations futures à répondre aux leurs.

S'inspirant largement de la jurisprudence de la Cour Européenne des Droits de l'homme, VADIM JEANNE soutien que la protection de l'environnement fait partie intégrante de l'intérêt public. ${ }^{1}$ La biosphère étant un bien commun, capable de satisfaire les besoins d'un grand nombre de population mondiale et, en tant que tel, interpelle une conscience collective de toute la communauté internationale. Selon DORST (1978), l'avènement de la société industrielle, la transformation de la nature, s'est intensifiée de façon considérable par rapport aux siècles précédents. ${ }^{2}$ De ce fait, le travail humain constitue un facteur déterminant de la dégradation de ressources naturelles et biologiques. L'exemple donné par D. ETIENNE et D. TESTARD sur les mouvements écologistes est amplement révélateur de l'utilisation abusive de la notion de l'intérêt public. Ce processus ne signifie pas une surcapacité de l'idéologie dominante de l'intérêt public à résister aux attaques même les plus virulentes, mais montre que tout mouvement contestataire qui n'a pas un caractère organique, c'est-à-dire contenant un principe interne d'organisation qui ne viendrait pas de l'Etat. ${ }^{3}$ Cette idée est naturellement révélatrice de la notion de cogestion ou de la gestion des aires protégées à des finalités de la conservation dont les acteurs même privés sont associés à cette gestion ou cogestion. Sur abondamment, l'utilisation des ressources à des niveaux insoutenables et la contamination de l'environnement par la pollution et des déchets à des niveaux dépassant la capacité de l'environnement à les absorber ou à les rendre inoffensifs. L'impact des activités anthropiques sur l'environnement est généralement négatif. Ces activités consistent d'une part, en des prélèvements effectués dans la nature, et d'autre part en des rejets des déchets sous forme solide, liquide ou gazeuse. Le travail humain est donc la cause principale du recul de la biodiversité. ${ }^{4}$

GUY CANIVET pense que si tout le monde ne mène pas une action forte et à multiples facettes, la biosphère risque de devenir incapable de préserver la vie humaine. Tout au moins, les générations futures subiront des privations et des difficultés à moins que les modèles actuels de production, de consommation et de gestion des déchets ne subissent un changement radical. Il est nécessaire que le développement durable devienne le mot d'ordre

1 VADIM Jeanne, La protection de l'environnement, composante de l'intérêt général : étude du traitement jurisprudentiel de la notion par la Cour européenne des Droits de l'Homme, Mémoire Master 2, Faculté Jean Monnet, Droit, Economie, Gestion, Université Paris Sud, 2015, p. 13.

2 DORST cité par Kamal BOUSHABA, Relations sociétés-nature et stratégies intégrées de conservation et du développement : Cas de la Réserve de Biosphère Intercontinentale de la Méditerranée, Thèse, Université de Montréal, Faculté de l'Aménagement, Janvier, 2017, p. 54.

3 ETIENNE D. et TESTARD D., Les idéologies de l'environnement, Centre, Périphérie, Territoire, P.U.F., Paris, 1978, pp. 133-150.

4 KATOBO C. B., Exploitation minière, une menace pour les aires protégées du KATANGA cas de PHELPS DODGE CONGO A LA BASSE KANDO, PREMI CONGO, Kinshasa, 2013, p. 14. 
et la politique de tous les organismes publics et des dirigeants et que chaque personne s'en sente responsable. ${ }^{5}$

Le droit de l'environnement est un outil indispensable à la gestion et à l'administration du développement durable. Il prévoit la création de politiques gouvernementales et d'actions pour la défense de l'environnement, qui garantissent l'usage à la fois équitable et durable des ressources naturelles. Le concept de conservation de la nature a près de 150 ans et ses fonctions ainsi que les outils mis de l'avant pour arriver à ses fins ont bien évolué depuis. Dès Déjà vers les années 1970 , la création de grandes organisations mondiales a marqué le début d'une prise de conscience collective liée à l'importance de conserver la nature, se justifie par la nécessité de l'intérêt public. L'intérêt public, tout en reconnaissant que l'appareil d'Etat n'a pas l'exclusivité de sa perception, mais l'Etat serait bien le maître et l'utiliserait à sa guise sans que cette notion puisse s'opposer à son activité ou constituer une limite à son action : l'efficacité de l'intérêt public serait alors pratiquement absolue. ${ }^{6}$ Ce qui fait que l'on assiste actuellement à une recrudescence des acteurs souvent non officiels, intervenants dans le domaine de la conservation et de la protection de l'environnement.

En RDC, les politiques publiques relatives à la conservation de la nature avaient été matérialisées à travers l'Ordonnance - loi nº6-041du 22 Août 1969 relative à la conservation de la nature et ses mesures d'application. Faute des mesures d'application et de certaines dispositions jugées lacunaires de l'Ordonnance - loi susmentionnée, son exécution s'est avérée difficile. ${ }^{7}$ C'est dans ce contexte que le pays s'est vu dans la nécessité de se doter d'un nouveau cadre juridique, à savoir la loi n $14 / 003$ du 11 février 2014 relative à la conservation de la nature en RDC afin d'adapter les dispositions légales aux enjeux de l'heure sur le droit de la conservation de la nature.

Ce nouveau cadre légal a innové sur plusieurs aspects dont les plus significatifs restent la définition des mesures générales de conservation, de la diversité biologique et de l'utilisation de ses éléments constitutifs, l'implication de la Province et de l'Entité Territoriale Décentralisée (ETD) à la conservation de la nature. En vertu de ses prérogatives discrétionnaires, l'Etat peut décider de manière unilatérale aux différentes mesures de protection et de conservation de la nature, le cas échéant déroger à ces mesures lorsque les nécessités de l'intérêt public l'exigent. Outres ces principes prévus par le nouveau cadre légal, il y a lieu de relever d'autres contenus dans les autres instruments juridiques pertinents nationaux et internationaux en la matière. C'est dans ce contexte que se situe la préoccupation centrale de cet article qui aborde la question autour de l'utilité de l'intérêt public, à travers son sens, sa compréhension, son contenu et son avenir dans le domaine du droit de la conservation de la nature en RDC. Pour ce faire, la présente étude a fait usage à la méthode exégétique et à

5 CANIVET G., LAVRYSEN L. \& GUIHAL D., Manuel judiciaire de droit de l'environnement, Programme des Nations unies pour l'environnement, Nairobi, 2006, p. 2.

6 CHEVALLIER J., L'intérêt général dans l'administration française, pp. 327 et 33.

7 Préambule de la loi $n^{\circ} 14 / 003$ du 11 Février 2014 relative à la conservation de la nature en République Démocratique du Congo. 
la sémiotique juridique pour décrypter le sens du concept intérêt public, son interaction multidimensionnelle dans le contexte de la gouvernance des aires protégées et des intérêts multiples de l'Etat et des collectivités territoriales.

\section{Plan sommaire}

De toute cette préoccupation, il se dégage une nécessité à la fois de la compréhension du concept intérêt public (A), le lien qu'il entretient avec la conservation de la nature (B), de son encadrement $(\mathrm{C})$ et l'avenir de la conservation de la nature en RDC au travers le concept d'intérêt public (D).

\section{A. Enigme de la compréhension de l'intérêt public}

L'intérêt public ou mieux l'intérêt général fait partie de nombreux concepts juridico-politiques qui sont indéfinissables. ${ }^{8}$ Sans prétendre à l'exhaustivité de son contenu, qu'il s'agisse de l'intérêt public, de l'intérêt général, de l'intérêt social, de l'intérêt national ou de l'intérêt commun; tous aspirent exprimer qu'une idée, celle orientée vers les finalités de bien-être de la communauté ou du bien commun Bien que ces concepts expriment en apparence une idée simple, ils prennent cependant des perceptions variables selon le contexte et les individus qui les emploient, l'intérêt public en est donc un exemple frappant, sans substance fixe. Même si l'on se limitait à son emploi juridique, il faut reconnaître que ce concept n'a jamais eu de définition claire et stable.

La notion de l'intérêt public est immense, ce qui lui confère le caractère amovible, versatile interdisciplinaire, polysémique voire mou, rendant ainsi son analyse et sa compréhension très complexe. Associer cette notion d'intérêt public aux réalités et à l'évolution du droit de la conservation de la nature en RDC, vient une fois renforcer l'ambigüité de sa compréhension. Face à cette affluence des significations possibles rattachées à ce concept d'intérêt public d'une part, et les évidences de la conservation de la nature en RDC, il est souhaitable de se poser la question de savoir s'il est possible d'envisager une compréhension précise de cette notion dans le cadre du droit de la conservation de la nature en RDC, et si cette notion a réellement un sens dans l'avenir de la conservation de la nature.

\section{B. Encadrement de l'intérêt public}

Prenant en considération toutes les ambiguïtés ci-haut suscitées par le concept d'intérêt public, il parait propice de se fixer sur cette notion afin de bien l'appréhender dans le contexte concret de cette étude. Ainsi, parler de l'encadrement de l'intérêt public, c'est comprendre tout d'abord sa notion (I), puis avancer sa vocation constituante (II), ensuite ses fonctions (III), ses critères (IV) et enfin ses limites (V).

8 CYRIAQUE L., et al., Contribution à l'analyse de l'idéologie de l'intérêt général, Université d'Amiens, discours et idéologie, 1966, p. 2. 


\section{Notion d'intérêt public}

La question de la définition de l'intérêt dit (public) est souvent confondue avec la question de son contenu, ce qui ne parait pas bon à notre avis de procéder par avance à toute possibilité de définition. Toutefois, cela ne signifie pas tout principalement que l'intérêt public est idéologique et qu'il remplit aussi une fonction théorique et technique au sein du droit. Par contre, l'intérêt public n'est pourtant pas isolable. Elle occupe une place qui est, prioritairement, celle de la légitimation au sein de l'idéologie dominante. ${ }^{9}$ Il semble difficile d'agencer précisément cette place, dans la mesure où la notion de l'intérêt public imprègne toutes les instances des autres domaines de la vie humaine, notamment le droit, la politique, la philosophie et, se répercute par-là sur l'infrastructure. C'est pourquoi, il ne paraît possible de l'assimiler à un domaine strictement juridique.

Le sens de l'intérêt public est défini et exercé par le pouvoir politique principalement le gouvernement de la républuque, dès lors que celui-ci établi son action gouvernementale. De ce fait, l'intérêt public est rattaché automatiquement à l'Etat ou il est identifié à la nation. La notion d'intérêt public revêt une acception politique et juridique. Considéré du point de vue politique, l'intérêt public n'est pas la somme des intérêts particuliers. Cependant, il ne faut pas penser que l'intérêt public n'a rien avoir avec les individus ou les groupes composant la nation; il a pour bénéficiaires finaux les hommes nés ou à naitre. Le sacrifice de l'intérêt individuel ou particulier est le sacrifice d'hommes pour d'autres hommes. L'intérêt public n'est pas distinct, par essence de celui des personnes ou des groupes. Il est un arbitrage entre les divers intérêts particuliers : l'exemple patent est l'expropriation qui a pour objet le passage d'une autoroute sacrifie l'intérêt du propriétaire à celui des innombrables usagers de la route. Il est parfois défini qualitativement, et non quantitativement. Tout comme les malades indigents ne constituent pas les groupes le plus nombreux de la population mais la valeur de la santé et de la vie humaine l'emporte sur celle des intérêts pécuniaires de l'ensemble des citoyens, justifiant l'existence d'une assistance médicale gratuite aux frais des contribuables. Suivant les pays et les époques, le critère quantitatif et, surtout le critère qualitatif prédominent.

Autre chose, la notion d'intérêt public varie dans le temps et dans l'espace. Du point de vue juridique, pour que la notion d'intérêt public soit utilisable, les autorités ayant compétence pour arbitrer entre les intérêts particuliers doivent être déterminées. Le constituant définit parfois lui-même l'intérêt public. C'est ainsi que le préambule de la constitution française de 1946 met au rang des intérêts publics la santé, la sécurité des travailleurs, la protection de la famille, de la femme et de l'enfant. ${ }^{10}$ En règle générale, pourtant le législateur a compétence pour définir la plupart des éléments composant l'intérêt public, avec la précision nécessaire, mais il se peut que ce dernier ne procède pas à une clarification de cette notion laissant ainsi une interprétation divergente. Toujours en France, la loi du 5 avril

9 CYRIAQUE L., et al., op. cit., p. 8.

10 Constitution de la République Française de 1945. 
1884 définit l'ordre public, catégorie de l'intérêt public, comme la sauvegarde de la tranquillité, de la sécurité et de la salubrité publique. ${ }^{11}$ En créant un service public, le législateur brande l'objet de ce service au nombre des éléments de l'intérêt public. En général, pourtant le législateur a compétence pour définir la plupart les éléments composant l'intérêt public, avec la précision nécessaire. Ainsi, il définit également les règles juridiques à respecter afin de poursuivre la fin de l'intérêt général. L'autorité compétente pour intervenir en la matière et les moyens dont elle pourra user.

L'administration quant à elle, est compétente pour définir les fins de l'intérêt public dans le domaine qui n'est pas réservé au législateur. Il lui appartient, notamment, de définir tel ou tel aspect particulier de l'intérêt public, dans le respect de la loi et des principes généraux du droit. ${ }^{12} \mathrm{~A}$ notre avis, l'intérêt public est l'avantage qui profite à tous et non à un individu bien déterminé. Ainsi, dans le cadre du droit de la conservation de la nature, l'intérêt public apparait à la fois en tant qu'élément régulateur et en tant que protecteur. Entant que régulateur, l'intérêt public garantit les intérêts collectifs liés aux ressources naturelles et biologiques. Par contre, en tant qu'élément protecteur, l'intérêt public permet très souvent de restreindre les droits et libertés individuelles au détriment de la communauté présente afin de permettre à cette dernière de tirer profit de ces ressources naturelles et biologiques tout en les préservant rationnellement envie de permettre aux générations futures d'en bénéficier. Toutefois, lorsqu'une politique, une pratique ou une institution est dans l'intérêt public c'est lorsqu'elle concourt à l'intérêt, à l'avantage, au bienfait ou pour le bien d'un groupe particulier ou d'un ensemble de gens faisant partie de ce groupe. De ce fait, il y a lieu de comprendre implicitement que l'intérêt public existe tant national, provincial et local selon qu'il relève de la compétence du pouvoir central, provincial et local.

\section{Distinction entre intérêt public et intérêt particulier}

La différence entre l'intérêt public et particulier n'est en effet qu'apparente. Elle masque et révèle à la fois un rapport cohérent entre acquiescement qu'il y a d'une part entre la compression de cette notion et de l'autre le conflit existentiellement originaire de l'intérêt public et de l'intérêt particulier. Une certaine franche de la doctrine notamment libérale soutient que l'intérêt public est la somme consensuelle d'intérêts particuliers et même comme le dépassement de ces intérêts. Cette doctrine soutient en outre l'existence irréductible et nécessaire d'intérêts particuliers conflictuels et l'existence d'une harmonie fondamentale entre l'intérêt public et les intérêts particuliers. ${ }^{13}$

La notion d'intérêt public repose tout d'abord sur une idée d'existence d'intérêts particuliers, définis à priori comme contradictoires puisque visant à promouvoir un avantage

11 ROUAULT M. C., Droit administratif et institution administrative, Larcier, Paris, 2013, p. 345.

$12 A M B L A R D C$., Intérêt général, utilité publique ou utilité sociale : quel mode de reconnaissance pour le secteur associatif? XXII ${ }^{\text {ème }}$ Colloque ADDES, Paris, 2009, p. 32.

13 GUYOMAR J.Y., L'idéologie nationale, éd. du Champ libre, 1974, p. 216. 
personnel. Tandis qu'une autre affirmation de cette notion de l'intérêt public soutient l'hypothèse selon laquelle, serait le dépassement des intérêts publics et qui assurent la conciliation de ces intérêts dits particuliers. ${ }^{14}$

\section{Superposition de l'intérêt public}

Dès lors que des collectivités exercent un certain nombre de compétences, elles font des choix en fonction de l'intérêt général du territoire dont elles ont la charge. Il n'est donc pas absurde de parler d'intérêt général régional ou local. Si l'on considère que la définition de l'intérêt général et les mesures d'application qui en découlent constituent l'essence même du politique, les différents niveaux d'intérêt général sont régis par des règles qui définissent les relations entre les différents niveaux de collectivités publiques, avec une hiérarchie des niveaux en fonction d'un niveau d'intérêt général supérieur ${ }^{15}$. En toute évidence, il existe un chevauchement de l'intérêt public qui part de l'échelle mondiale jusqu'à la base au niveau de la base ou local. De cette hiérarchisation échelonnée de cette notion, il est tout à fait logique de le définir clairement selon le cadre juridique propre aux autorités compétentes. S'agissant justement de cet intérêt public mondial, dans le contexte du droit de la conservation de la nature, l'environnement et les ressources naturelles et biologiques sont considérés comme le bien public de toute l'humanité. C'est dans cette optique que le droit international est parvenu à consacrer la notion du patrimoine commun de l'humanité. Nombreux sont les aires protégées de la RDC qui sont classées parmi les patrimoines communs de l'humanité.

Dans le contexte de l'intérêt public à l'échelle nationale, comme évoqué dans les lignes qui précèdent, relève de la compétence de l'Etat ou du pouvoir national. En effet, à titre originaire, le monopole de la définition de l'intérêt public revient au pouvoir discrétionnaire du gouvernement, qui doit donner le sens et le contenu de cet intérêt public à travers sa politique et son action gouvernementale. Le régionalisme politique que consacre la constitution de la RDC du 18 février 2006 telle que modifiée et complétée à ces jours ainsi que les dispositions légales pertinentes, révèlent l'existence de la superposition de pouvoir étatique. Ces dispositions établissent une répartition des compétences entre le pouvoir central, la province et l'ETD. Parmi ces compétences, il y a celles qui sont exclusives et celles qui sont concurrentes. C'est ainsi que la province et l'ETD se trouve intéresser par la conservation de la nature en RDC, car elles sont des entités politiques dotées des compétences propres.

Par contre, faisant allusion au droit de la conservation de la nature, les compétences entre ces niveaux de pouvoir public ne sont pas clarifiées pour permettre une efficacité de la conservation de la nature en RDC. A titre indicatif, cette imprécision se situe principale-

14 FENET A., «La question des minorités, propos sur la souveraineté », in Réalités du droit internationalcontemporain, Reims, 1976, p. 205.

$15 L U C A S J .-M$., ESS et intérêt général sous le regard de l'Union européenne, octobre 2012. Voir $\mathrm{http} / / / \mathrm{www}$.lartestpublic.fr/ressources/ressources-documentaires/125-ouvrages-thematiques/321-es s-et-interet-general-sous-le-regardde-l-union-europeenne-jean-michel-lucas-octobre-2012. 
ment au niveau de la prise des mesures dérogatoires aux principes de la conservation de la nature prévue par la loi de 2014. Ces mesures dérogatoires prises dans l'intérêt public peuvent être soit de la compétence exclusive de la province soit de l'ETD, étant donné qu'il y a existence d'un intérêt public provincial ou local. La dimension de la conservation de la nature doit en effet être partagée et concertée entre les institutions internationales de la conservation, l'Etat congolais et voire les acteurs non étatiques afin de permettre une bonne conservation de la nature.

\section{Contenu de l'intérêt public}

La dimension intrinsèque du concept intérêt public se caractérise par une certaine flexibilité. C'est donc une notion mouvante et se présente souvent différemment selon les contextes utilisés. L'on doit cependant s'accorder que ce concept d'intérêt public est dépourvu de contenu intrinsèque universel. Toutefois, on ne peut comprendre la fonction et les effets de cette notion que si on essaye de préciser le contenu de son imprécision. Dans cette considération, l'intérêt public est souvent présenté comme la finalité du droit public ou de l'appareil d'Etat. Mais cette notion demeure toujours inachevée, car autant l'individu se pose la question de la finalité de l'Etat et de la société, autant il en fait pour ce qui est de l'intérêt public.

La doctrine et la jurisprudence administratives paraissent reconnaître aujourd'hui l'existence de plusieurs intérêts publics : l'intérêt public économique, l'intérêt public social, l'intérêt public culturel. Ces divers intérêts publics généraux peuvent être, sinon conflictuels, du moins difficilement compatibles en certaines circonstances. Le contenu de l'intérêt public est à la base de la refondation, de la résolution du conflit originel entre la liberté de l'individu et la liberté de tous. Dans le premier cas, l'intérêt public constitue un élément conciliateur entre les libertés individuelles. On pourrait alors aussi dire que l'intérêt public est la chose publique, par conséquent celle-ci n'est rien d'autre que l'État lui-même. L'État est fait, non pas de la somme d'intérêts particuliers mais seulement de la somme de tous les intérêts que les hommes ont décidé, par un acte libre de volonté, de mettre en commun.

La doctrine libérale soutient à la fois l'existence irréductible, nécessaire d'intérêts particuliers-conflictuels et, l'existence d'une harmonie fondamentale entre l'intérêt général et les intérêts particuliers. L'intérêt public ne peut pas être défini, dans son contenu, simplement comme l'apanage de l'Etat. En effet, cela conduirait à l'idée que l'intérêt public n'a d'autre contenu que celui que l'Etat lui donne. Cependant, dans l'état actuel de la conservation de la nature, il s'observe une multiplicité d'acteurs étatiques nationaux et internationaux ou non, qui participent d'une manière au sens intrinsèque du concept de l'intérêt public dans le cadre de la conservation de la nature en RDC. Dans cette optique, la notion d'intérêt public ne constituerait en aucune manière une limite à la politique et à l'action de l'Etat. C'est ainsi que les partis politiques, les syndicats, les groupes de pression jouent ce rôle et donnent aussi un sens au contenu de l'intérêt public. Ces diverses organisations, même si leur mission semble, pour certaines, opposée à celle des gouvernants, ont aujourd'hui remarquablement intégré le contenu de l'intérêt public. 


\section{Fonctions de l'intérêt public}

1. Fonction politique de l'intérêt public

Dès que la politique a été perçue comme une idéologie, c'est-à-dire dès que les individus vivant en communauté ont cherché à expliquer et légitimer leur mode d'organisation politique, le thème de l'intérêt public a été présent. L'intérêt public comme nous l'avions soutenu précédemment, remplit les fonctions traditionnelles de l'Etat, et par là elle tend à légitimer l'action de ce dernier en assurant la reproduction de l'ordre social.

La fonction primordiale que remplit la notion de l'intérêt public est éminemment politique en ce que, considéré sous cet angle politique, l'intérêt public parait comme un outil au service de l'Etat. Or, l'intérêt public est défini et exercé par l'Etat dans le cadre de ses attributions traditionnelles liées à sa souveraineté. De surcroit, l'action étatique est justifiée par les exigences de l'intérêt public. Pour mieux donner le sens de la fonction de l'intérêt public sous cet aspect étatique, il faut utiliser l'approche consistant à penser l'intérêt public au-delà du phénomène de dilution de la notion. Dans ce cas la notion n'est plus à considérer comme dépourvue de sens, mais plutôt par les fonctions régaliennes de l'Etat ${ }^{16}$.

S'il faut rentrer dans le cadre de cet article, il parait clair qu'en matière de conservation de la nature, les autorités étatiques au niveau national, provincial ou local définissent les politiques relatives aux mesures de gestion, de protection et de conservation de la nature en RDC, posent les principes et éventuellement dérogent à ces principes, chacunes dans les limites de leurs compétences,

\section{Fonction administrative de l'intérêt public}

Dans sa perspective purement administrative, l'intérêt public est considéré comme critère de détermination de service public. L'intérêt public légitime l'action de l'Etat. Celui-ci, pour parfaire son action, recourt à son appareil de fonctionnement à savoir l'administration. Ainsi, l'administration s'assure très souvent de la présence du critère d'intérêt public pour savoir si une activité donnée est conduite dans l'intérêt public ${ }^{17}$. Au-delà de cet aspect qui tient au contenu de l'intérêt public, l'Etat met toujours en place un organisme public chargé de garantir cet intérêt public. Dans le cas sous examen, l'Institut Congolais de Conservation de la Nature (ICCN) qui est un organisme public vient concrétiser cette fonction administrative de la notion de l'intérêt public.

16 SIMARD N.D., Pour un élargissement de la notion d'intérêt public, ADEC, 2003. p. 73.

17 TIGER N., L'intérêt général et le juge administratif, in Rapport public du Conseil d'Etat, Paris, 1999 , p. 9. 


\section{Fonction économique de l'intérêt public}

Le but de l'activité de l'intérêt public est la satisfaction de bien commun. De cette notion de bien commun on voit que la mission de l'intérêt public doit être intéressé ou non rentable. Le cas le plus évident c'est que l'intérêt public a comme contenu le bien commun. Celui-ci bénéficie d'un certain nombre de principes à savoir la gratuité dont le désintéressement par rapport à la poursuite d'un quelconque profit. Il est inconcevable de parler de l'intérêt public sans traiter la question de bien commun, qui est le critère général de l'équilibre de la notion de l'intérêt public. Le bien commun n'est pas la somme des biens individuels mais un bien spécifique qui se situe au-delà des biens individuels. Le bien commun réfère à des valeurs et des intérêts partagés et publics. Il oriente vers la réalisation d'objectifs partagés comme éléments normatifs et décisifs de la vie sociale et comme condition d'une vie digne de l'être humain et de tout être humain. Les ressources naturelles et biologiques que regorge la RDC sont des biens publics dans la mesure qui profitent à tout le peuple congolais. La fonction économique de l'intérêt public confère au bien public le caractère de bien ou de patrimoine commun de l'humanité au niveau mondial et qui justifie la protection des espèces de faune et de flore à l'échelle nationale, provinciale et locale.

\section{Fonction sociale de l'intérêt public}

Une autre fonction que remplit l'intérêt public est celle d'assurer la cohésion sociale. De ce fait, l'intérêt public tend à occulter et denier les systèmes de domination des classes sociales. On assiste aujourd'hui à un double mouvement de désétatisation de l'intérêt général et de technicisation de l'intérêt public. La désétatisation de l'intérêt public s'entend comme une manière dont l'Etat n'a plus le monopole de la définition et du sens de l'intérêt public.

A ces jours, certains acteurs non étatiques, nationaux ou internationaux orientent leurs actions vers les missions de l'intérêt public. Alors que la technicisation de l'intérêt public, se substituant au mythe monstrueux de l'Etat-Léviathan, extérieurs aux individus, apparaît l'image de l'Etat-arbitre, calculateur rationnel et scientifique des intérêts particuliers ${ }^{18}$. L'intérêt général perd alors sa connotation légendaire pour prendre une connotation économique. L'intérêt public est constitué de l'ensemble des besoins de la communauté qui sont distincts de ceux des invendus qui composent cette société.

\section{Fonction scientifique de l'intérêt public}

La science représente actuellement un facteur très important dans le domaine de l'environnement en général et de la conservation de la nature. Ainsi, les expérimentations et prélèvements sur certaines espèces végétales et biologiques sont autorisés pour l'intérêt général fondé sur l'avancement de la science.

18 DELECOURT B., Intérêt Social, Mémoire D.E.A, Droit des contacts, Université de Lille II, 2001. 


\section{Fonction culturelle de l'intérêt public}

La culture en tant que mode de vie d'un peuple constitue un support auquel toute action ou politique gouvernementale doit tenir compte pour répondre aux attentes de sa population. Toutes ces fonctions énumérées ci-haut sont loin d'être exhaustives compte tenu de l'étendue que la notion d'intérêt public peut se mesurer. Pour démontrer à suffisance le caractère transversal et interdisciplinaire de cette notion d'intérêt public, cette étude a voulu épingler quelques-unes d'entre elles. C'est dans ce cadre que le droit international accorde une importance capitale au patrimoine culturel en le considérant comme un bien public auquel consommé par tous les peuples du monde en quantité égale et ce, en dehors de la satisfaction du principe de concurrence.

\section{Critères de l'intérêt public}

En droit, la notion de l'intérêt public est souvent employée dans le cadre du droit administratif, principalement dans les services publics, ce qui sous-tend créer un lien direct entre cette notion et celle de la personne publique ${ }^{19}$. C'est dans ce sens que l'intérêt public est souvent pris comme critère pour définir le service public en dépit de la difficulté que suscite ce concept du fait de son inamovibilité. Toutefois, pour juger une activité d'intérêt public ou général, il faut se baser sur certaines considérations purement d'ordre théorique. Il en est ainsi de la distinction entre l'intérêt public et l'intérêt particulier ou individuel et même la somme de ces intérêts. En effet, l'intérêt public dépasse de loin ces intérêts et s'impose à eux, au nom du bien commun. Concrètement le critère de l'intérêt public est évidemment reconnu aux activités qui touchent aux fonctions régaliennes de l'Etat comme la sécurité publique, la défense, la justice. En revanche l'application de ce critère se pose dans tous les autres domaines dans la mesure où il n'y a pas d'activités d'intérêts publics par nature ${ }^{20}$.

\section{Limites de l'intérêt public}

L'évolution du droit de la conservation de la nature ne permet plus de soutenir le postulat traditionnel selon lequel l'Etat dispose du monopole de la définition et de l'activité de l'intérêt public. Il faut actuellement recourir à une approche multidimensionnelle incluant l'ensemble d'acteurs environnementaux à la détermination du contenu effectif de la notion de l'intérêt public. On assiste aujourd'hui à un double mouvement d'une part de désétatisation de l'intérêt public à l'instar de la doctrine néolibérale qui prône le désengagement de l'intérêt public au profit de l'intérêt particulier; et d'autre part de technicisation de l'intérêt public qui est une approche nouvelle de la notion d'intérêt public pour être efficace, doit être dy-

19 COULET $W$., La notion de service public dans le droit de l'environnement, Mél. Montané de La Roque, Paris, 1988, p. 14.

20 CYRIAQUE L., et al., Contribution à l'analyse de l'idéologie de l'intérêt général, Université d'Amiens, discours et idéologie, 1966, p. 2. 
namique. Les tempéraments à apporter à la notion de l'intérêt public sont principalement liés à l'action de l'Etat. Rappelons que l'intérêt public est au service de l'Etat qui a la charge de le définir et de le donner un sens, conformément aux principes de sa souveraineté. C'est de là qu'il faut commencer à décrypter les limites de l'intérêt public.

En effet, la souveraineté étatique n'est plus actuellement le seul domaine réservé de l'Etat. C'est ici l'occasion de rappeler que la RDC n'exerce plus sa souveraineté de manière absolue, celle-ci est limitée par le droit international d'origine coutumière ou conventionnelle. En vertu de ces instruments juridiques internationaux conventionnels ou non-conventionnels, auxquelles elle est partie, la RDC est tenue s'y conformer au nom du principe de pacta sunt servanda. L'autre tempérament à apporter à la notion d'intérêt public découle du libéralisme qui prône les intérêts particuliers au détriment de l'intérêt public.

\section{Corrélation entre intérêt public et conservation de la nature}

Afin de mieux cerner le rapport qui existe entre la notion d'intérêt public et celle de la conservation de la nature, il est souhaitable de préciser tout d'abord les principes de protection et de conservation de la nature en RDC (I), ensuite les dérogations auxdits principes (II) et enfin le rapport d'usage qu'entretiennent ces deux concepts (III).

\section{Principes de la conservation de la nature en RDC}

Les dispositions de la loi relative à la conservation de la nature en RDC, réaffirment certains principes faisant référence à l'intérêt public. Parmi ces dispositions, il y a lieu de faire cas des articles 3, 9, 14 et 17. L'article 3 détermine la souveraineté de la RDC sur ses ressources naturelles et biologiques autant qu'elle le fasse sur sa population et son territoire. Ces prérogatives étatiques constituent le fondement de son action et qui s'oriente vers la satisfaction des besoins collectifs de son peuple.

Par ailleurs, l'article 9 énumère certains éléments constitutifs de la diversité biologique que l'Etat doit identifier. Ces éléments sont entre autres:

- Les écosystèmes et habitats comportant une forte diversité des espèces endémiques ou menacées, ou des étendues sauvages nécessaires pour les espèces migratrices;

- Les espèces et communautés menacées, d'intérêt médical, agricole ou économique, d'importance sociale, scientifique ou culturelle ou d'un intérêt pour la recherche sur la conservation et l'utilisation durables de la diversité biologique;

- Les génomes et gènes décrits revêtant une importance sociale, scientifique ou économique.

La loi charge donc l'organisme public qui est l'ICCN de procéder à la mise en œuvre de cette identification de ces éléments avec le concourt des autres acteurs intervenants dans cette matière. 
En ce qui concerne l'article 14 de ladite loi, il procède à l'interdiction de:

1) prélever, chasser, pêcher, capturer, harceler ou tuer délibérément des spécimens des espèces protégées;

2) perturber intentionnellement ces espèces, notamment durant la période de reproduction, de dépendance, d'hibernation ou de migration;

3) détruire, endommager, enlever, ramasser les œufs de ces espèces ou en modifier la position;

4) détériorer ou détruire les sites de reproduction, les aires de repos ou tout habitat naturel où vivent ces espèces à un des stades de leur cycle biologique;

5) détenir, transporter, échanger, vendre ou acheter, offrir ou céder à titre gratuit les spécimens ou toute partie de ces espèces prélevés dans la nature;

6) détenir, céder, vendre, acheter ou transporter tout produit dont l'emballage ou la publicité annonce contenir des spécimens appartenant à l'une des espèces protégées;

7) exposer dans les lieux publics ces spécimens.

Quant à l'article 17, il va dans le même sens en interdisant de:

1) couper, déraciner ou détruire intentionnellement des spécimens des espèces de flore menacées dans la nature;

2) détenir, transporter, vendre ou acheter des spécimens de ces espèces de flore prélevées dans la nature;

3) détériorer ou détruire intentionnellement les habitats dans lesquels la présence de ces espèces de flore est établie.

Ces principes prévus dans la loi relative à la conservation de la nature ne suffisent pas, encore faut-il recourir à d'autres instruments juridiques tant nationaux qu'internationaux pour dégager les autres principes liés à la conservation de la nature en RDC. ${ }^{21}$

\section{Les instruments juridiques nationaux}

C'est ainsi que nous retrouvons les dispositions éparses de ces principes dans le code des investissements, le code forestier, le code minier, la loi des hydrocarbures, la loi portant principes fondamentaux relatifs à l'agriculture et la loi relative à l'eau.

Loi $n^{\circ} 004$ du 21 février 2002 portant code des investissements: l'article 8 conditionne l'admission des investissements au régime général aux conditions de s'engager à respecter la réglementation en matière de la protection de l'environnement et de la conservation de la nature. L'article 31 point 5 parle du respect de la réglementation en matière de protection de l'environnement et de la conservation de la nature.

21 PRIEUR Michel, Les principes généraux du droit de l'environnement, cours, Master 2, Faculté de Droit et des Sciences Economiques, Université de Limoges, Agence Universitaire de la Francophonie, Limoges, 2003, p. 16. 
Loi $n^{\circ} 011 / 2002$ du 29 Août 2002 portant code forestier: cet instrument met en place un certain nombre des principes de gestion de ressources forestières. L'Etat a l'obligation d'élaborer une politique forestière nationale matérialisée par un plan forestier national à réviser périodiquement en fonction de la dynamique de l'industrialisation forestière. Le code forestier distingue trois catégories des forêts à savoir les forêts classées, les forêts protégées et les forêts de production permanentes qui sont soustraites des forêts protégées à la suite d'une enquête publique en vue de leur concession. Pour assurer le développement durable des ressources naturelles, la loi introduit dans la gestion forestière deux concepts celui d'inventaire forestier et celui d'aménagement forestier. Par ailleurs les forêts classées sont celles soumises en application d'un acte de classement, à un régime juridique restrictif concernant les droits d'usage et d'exploitation, elles sont affectées à une vocation particulière notamment écologique. L'article 25 reconnait que le Ministre peut, par arrêté, déléguer en tout ou en partie, la gestion de forêts classées à des personnes morales de droit public ou à des associations reconnues d'utilité publique dans le but de les protéger et de les mettre en valeur et d'y conduire les travaux de recherche ou d'autres activités d'intérêt public.

Loi $n^{\circ} 007 / 2002$ du 11 juillet 2002 portant code minier : il commence avec la réaffirmation du principe de l'exclusivité, de l'inaliénabilité et d'imprescriptibilité des gîtes des substances minérales, y compris les gîtes artificiels les eaux souterraines et les gîtes géothermiques se trouvant sur la surface du sol ou renfermés dans le sous-sol ou dans les cours d'eaux du territoire national. Les dispositions des articles 202 à 204 abordent d'une part de la protection de l'environnement et de l'étude à impact environnemental. L'article 291 dispose que l'annulation des droits miniers ou de l'autorisation d'exploitation de carrière permanente n'a pas pour effet de dégager le titulaire de ses obligations environnementales.

Loi $n^{\circ} 15 / 012$ du 1 er août 2015 portant régime général des hydrocarbures : les grandes innovations de cette loi est qu'elle a mis en place les principes de la création de la société d'hydrocarbure la création d'un fond en faveur des générations futures et le renforcement de la protection de l'environnement. L'article 11 donne pouvoir au gouvernement d'élaborer et de mettre en œuvre la politique nationale en matière d'hydrocarbures. Tandis que l'article 19 institue une création de fond pour les générations futures. Pour sa part, l'article 58 alinéa 8 parle d'une étude à impact environnemental et social préalable assorti de son plan de gestion dument approuvé qui doit être figuré parmi les autres éléments du plan de développement et de production. L'article 155 stipule que, l'exercice des activités d'hydrocarbures en amont est interdit dans les aires protégées et les zones interdites. Les articles 156, 157 et 158 ainsi que les articles 162 et 163 abordent dans le même sens les mécanismes de protection de l'environnement dans le cadre de l'exploitation des activités d'hydrocarbures.

Loi $n^{\circ} 11 / 022$ du 24 décembre 2011 portant principes fondamentaux relatifs à l'agriculture:

L'article 5 reconnait la prérogative à l'Etat, la province et à l'entité territoriale décentralisée d'adopter une approche intégrée de la conservation, de la prospection, de la collecte et de l'utilisation durable des ressources psychogénétiques pour l'alimentation et l'agriculture. 
L'article 6 par contre reconnait au gouvernement le pouvoir de définir et de mettre en place une politique agricole nationale en vue de la promotion et de la croissance de la production agricole. L'article 47 point b accorde à l'Etat et la province, dans les limites de leurs compétences respectives, le privilège de prendre des mesures destinées à l'utilisation des produits phytosanitaires sans danger pour l'environnement et la santé. Les articles 66 à 71 parlent de la protection de l'environnement que les exploitants forestiers sont tenus de respecter.

Loi $n^{\circ} 11 / 009$ du 09 Juillet 2011 portant principes fondamentaux relatifs à la protection de l'environnement : L'article 3 fait de l'environnement un patrimoine commun de la nation sur lequel l'Etat exerce sa souveraineté permanente. Sa gestion et sa protection sont d'intérêt général et elles sont soumises au respect du principe de développement durable. De ces différentes dispositions légales relatives à la conservation de la nature en RDC, deux situations évidentes se dégagent. D'une part, ces dispositions abordent les questions relatives à la conservation de la nature que les acteurs concernés sont tenus de respecter l'environnement, les écosystèmes, les substances naturelles et biologiques qui s'y trouvent. D'autre part, l'analyse de ces dispositions éparses de droit de la conservation de la nature, démontre de manière implicite la notion d'intérêt public. En effet, de manière générale l'élaboration des politiques nationales tant par l'Etat, la province ou l'entité territoriale décentralisée et l'institutionnalisation de certains services publics font intervenir dans un sens comme dans l'autre la notion de l'intérêt public.

Loi $n^{\circ}$ 15/026 du 31 décembre 2015 relative à l'eau : l'eau est une ressource économique mais aussi un bien social car l'un des rôles essentiels de l'eau est la préservation de la vie. Les dispositions pertinentes de cette loi abordent des questions relatives à la gestion des eaux, des lacs, et des cours d'eaux. Elle fixe en outre les règles de la gestion durable et équitable des ressources en eau. A son article 12 ladite loi définit la politique de la nation en matière de gestion, protection et d'utilisation de ressources en eau.

2. Les instruments juridiques internationaux relatifs à la conservation de la nature

Reconnaissant le droit de la protection et de la conservation de la nature comme un droit transversalement fondamental de l'homme, il n'est donc pas évincé que l'intérêt public intervienne dans ce domaine afin de consacrer le caractère de principe universel s'imposant à tous. ${ }^{22}$ Ces instruments, qui ont une portée mondiale, fournissent à peu près partout sur la planète le cadre général de la protection. Ils peuvent être rangés dans deux catégories classiques relatives, d'une part, aux instruments non contraignants et, d'autre part, à ceux qui ont un caractère obligatoire et qui sont essentiellement conventionnels.

Pour ceux qui ne sont pas contraignants, ils se bornent à poser un ensemble de conceptions générales de la protection, notamment sous la forme de principes d'orientation de

22 NIRMAL Nivert, Intérêt général et droits fondamentaux, Thèse, Université de la Réunion, Faculté de Droit et de l'Economie, Centre de Recherche Juridique, 2012, p. 96. 
l'action ou de la réglementation et sous la forme de programmes, plans ou codes d'action concrète. Il en est ainsi de la Déclaration de Stockholm 1972, de la Stratégie mondiale de la conservation de la nature de 1980, de la Charte mondiale de la conservation de la nature de 1982, de la stratégie pour l'avenir de la vie de 1991, de la déclaration de Rio de 1991 ou encore de la déclaration sur les forêts de 1992. Tandis que les instruments juridiques internationaux qui ont un caractère obligatoire, il y a ceux qui envisagent des méthodes de conservation de portée globale comme la Convention sur le droit de la mer de Montego Bay du 10 décembre 1982, la Convention sur la diversité biologique de Rio de 1992 et la Convention sur la lutte contre la désertification de Paris 1992 et celle de porté particulière comme la convention relative aux zones humides d'importance internationale de Ramsar de 1971 et la convention pour la protection du patrimoine mondial, culturel et naturel de Paris de 1971.

$\mathrm{Au}$ niveau africain il y a la convention africaine sur la conservation de la nature et des ressources naturelles adoptée en 2003 à Maputo et dont la RDC a ratifié en 2008. Rappelons que cette convention n'est que la révision de la convention d'Alger adoptée le 16 Juin 1069 et dont le pays avait ratifié en 1976.

\section{Les dérogations aux principes de la conservation de la nature en RDC}

La loi en édictant les principes généraux de protection et de conservation de la nature, a aussi prévu des exceptions à ces principes. La préservation de la santé, de la sécurité publique, la recherche scientifique mais aussi la prévention des dommages à certaines activités humaines peuvent nécessiter d'intervenir sur des habitats et/ou des espèces protégés, causant selon le cas altérations, destructions et effarouchement.

Parce que les activités et projets indispensables à la société ou à l'environnement peuvent impacter l'état de conservation d'espèces de faune et de flore sauvages nationales vulnérables, l'octroi d'une dérogation à la protection stricte d'espèces et d'habitats est soigneusement contrôlé. Ces dernières sont prévues par les articles 19 et 20 de la loi de 2014 relative à la conservation de la nature. Les dérogations sont accordées soit par le gouvernement (1) soit par l'organisme public à savoir l'Institut Congolais pour la Conservation de la Nature (ICCN) (2).

\section{Les dérogations gouvernementales}

Les dérogations reconnues au gouvernement au travers le conseil des ministres, elles sont prévues par l'article 19 de la loi précitée. En effet, l'économie générale de cet article fait référence à l'intérêt public pour ainsi justifier la dérogation en vie de favoriser la recherche scientifique, et l'octroi de permis ou certificats d'exportation ou de réexportation spécimen d'une espèce de faune ou de flore sauvage intégralement, partiellement protégée. Comme l'on peut constater, cette dérogation touche directement aux principes de conservation de la nature. La dérogation ainsi accordée, n'est admise qu'à condition qu'il n'existe aucune 
autre solution satisfaisante et qu'elle ne nuise pas au maintien, dans un état de conservation favorable, des spécimens des espèces de faune et de flore concernées dans leur aire de distribution naturelle. En outre, cette dérogation doit avoir fait l'objet d'une étude d'impact environnement et que le plan y relatif doit faire objet d'une approbation.

\section{Les dérogations de l'ICCN}

S'agissant des dérogations de l'article 20 accordées par l'organisme public, elles sont admises qu'exceptionnellement pour des raisons suivantes:

- Dans l'intérêt de la protection de la faune et de la flore sauvages et de la conservation des habitats naturels;

- Dans l'intérêt de la santé et de la sécurité publique, ainsi que de la sécurité alimentaire des populations riveraines des aires protégées;

- Pour prévenir des dommages importants notamment aux cultures, à l'élevage, aux forêts, aux pêcheries, aux eaux et à d'autres formes de propriété;

- A des fins d'éducation, de repeuplement et de réintroduction de ces espèces et pour des opérations de reproduction nécessaires à ces fins, y compris la propagation artificielle des plantes;

- A des fins de recherche scientifique et de bio-prospection.

Ces dérogations sont soumises au régime d'information du ministre ayant en charge la conservation de la nature dans ses attributions. De l'analyse de ces dérogations légales aux principes de la protection et de la conservation de la nature, deux situations se dégagent. D'une part, les dérogations prévues à l'article 19 et accordées par le gouvernement, portent directement atteintes aux principes de conservation de la nature tels que consacrés par les dispositions des articles 3, 9, 14 et 17 de la loi sur la conservation de la nature. D'autre part, il existe des dérogations aux principes de la conservation qui sont accordées pour l'intérêt même de la conservation. C'est le cas des dérogations de l'institut national pour la conservation de nature contenues dans l'article 20 de la loi sus évoquée.

Déroger aux interdictions portant sur les espèces de faune et de flore sauvages protégées, dans le cadre d'activités ou projets bénéfiques à la société ou à l'environnement, requiert le respect d'une procédure exigeante, visant à s'assurer que ces opérations ne viendront pas perturber l'état de conservation des espèces concernées. Cela vise également à garantir que l'activité projetée répondra à l'objectif national et communautaire qui n'est rien d'autre que l'intérêt commun, celui de la préservation du patrimoine biologique.

\section{Utilité de l'intérêt public dans la Conservation de la nature}

La nature et toutes ses composantes de la RDC constituent le support inéluctable de tout le peuple congolais dont l'importance n'est plus à prouver. Cette visée des ressources naturelles et biologique du pays est aujourd'hui au centre de la préoccupation de l'Etat, des organisations nationales et internationales voire des individus. L'interpénétration existant 
entre la notion d'intérêt public et la conservation de la nature est évidente dans la mesure où le droit tant national et international érige en principes juridiques la protection et la conservation de la nature. C'est dans cette optique que l'on retrouve la notion d'intérêt public dans le droit environnemental en tant que principe général qui exprime la solidarité mondiale due à la globalité des problèmes d'environnement. En effet tous les peuples du monde ont des préoccupations communes liées à l'environnement traduisant une interdépendance permanente entre eux. De ce fait, il n'est donc pas exclu que la RDC emprunte les principes du droit de l'environnement énoncés par les normes internationales ou nationales. La consécration de l'intérêt public dans le cadre du droit de l'environnement en général et celui de la conservation de la nature en particulier renforce la transversalité de ce dernier en reconnaissant implicitement le droit de la conservation de la nature comme un droit fondamental de l'homme.

Le nouveau droit à l'environnement sain concerne les générations présentes. Mais au contraire l'irréversibilité de certaines atteintes du milieu naturel et aux espèces animales et végétales affecte nécessairement les générations future ${ }^{23}$. Aussi la décision publique ou privée doit systématiquement prendre en compte ses effets directs ou indirects sur le long terme. C'est dans cette optique qu'intervient la notion de développement durable laquelle notion exprime l'idée que les ressources vivantes ne doivent pas être ponctionnées à un tel point qu'elles ne puissent, à moyen ou long terme, se renouveler. Il faut donc garantir la perpétuation des ressources.

Cependant, toute politique ou décision de développement actuel doit s'assurer que l'action qui en découle de près ou de loin, ne portera pas préjudice ni aux générations futures, ni aux ressources communes comme l'eau, l'air, le sol, les espèces et diversités biologiques. L'environnement est d'intérêt public dans la mesure ou la protection des espaces naturels et des paysages, la préservation des espèces animales et végétales, le maintien des équilibres biologiques auxquels ils participent et la protection des ressources naturelles contre toutes les causes de dégradations qui les menacent préoccupent au plus haut point le pouvoir public. ${ }^{24}$ Toutefois, ce rattachement de la notion d'intérêt public à celle de la conservation de la nature se matérialise du point de vue juridique par le contrôle de la légalité, par l'institutionnalisation d'un service public de l'environnement et d'un ordre public écologique. ${ }^{25}$

\section{Le contrôle de la légalité}

La notion d'intérêt public dans le domaine de la conservation de la nature en RDC obéit principalement aux dispositions légales contenues dans la loi de la nature. En effet, outre le cadre juridique de portée universelle ou régionale relative à la conservation de la nature que

23 DEVILLE H., Economie et politique de l'environnement, Harmattan, Paris, 2011, pp. 14-18.

24 BINOT Aurélie, La conservation de la nature en Afrique centrale entre théorie et pratiques. Des espaces protégés à géométrie variable, Thèse, Université Panthéon-Sorbonne, Paris I, 2010, p. 34.

25 GENOT J.-C., Conservation de la nature : gérer les espèces ou les habitats, Athènes noctua en Bordure de Vosges du Nord, Alauda, Paris, 1993, pp. 61,181-194. 
la RDC est partie, l'instrument légal du pays reste la loi n¹4/003 du 11 février 2014 relative à la conservation de la nature en RDC.

\section{Le service public de la conservation.}

Dès lors que la protection ou la conservation de la nature est considérée comme d'intérêt général, il n'y a plus d'adversité à ce que les pouvoirs publics créent des services publics spécialisés chargés de le gérer. ${ }^{26}$ Bien avant l'avènement de la nouvelle loi sur la conservation de la nature, l'ordonnance loi de 1969 avait déjà institué un organisme public en l'occurrence l'Institut National pour la Conservation de la Nature (INCN) chargé de la protection et de la conservation de la nature en RDC. L'actuelle loi de 2014 institue à son tour l'Institut Congolais pour la Conservation de la Nature (ICCN). L'inventaire des textes juridiques nationaux qui abordent la problématique de la conservation de la nature en RDC, démontre à suffisance l'existence d'un service public selon le domaine établis.

L'ICCN est un établissement public à caractère scientifique et technique. Il jouit d'une personnalité juridique propre avec une autonomie de gestion financière et administrative. ${ }^{27}$ Parmi ses missions il y a celles:

- D'assurer la protection de la faune et de la flore,

- De valoriser la biodiversité en favorisant la recherche scientifique et en facilitant les activités d'écotourismes conformément à la législation en vigueur et dans le respect des principes fondamentaux de la conservation et,

- De réaliser ou de faire réaliser les études et assurer la vulgarisation à des fins scientifiques et didactiques dans le domaine de la conservation.

\section{L'ordre public écologique}

Il est certain que dans le domaine de l'environnement, il existe des règlements de police visant à interdire et à contrôler certaines activités humaines perturbatrices du milieu naturel. Comme pour toutes les règles de police, elles doivent légalement correspondre à la nécessité d'assurer les objectifs d'ordre public que sont traditionnellement la sûreté, la tranquillité et la salubrité publique. Ce qui implique une unité spéciale de la police chargé d'assurer l'ordre public écologique, de rechercher les infractions et de déférer les contrevenants devant les juridictions compétentes en matière de protection de l'environnement. Ainsi dans le cadre actuel de la conservation de la nature en RDC, la loi a quand même prévu un gardefou en créant une unité spéciale de la police chargée de la protection de la nature appelée les éco-gardes. 


\section{Avenir de la conservation de la nature en RDC au travers le concept d'intérêt public}

Dans ce dernier point, il sera question tout d'abord d'aborder les difficultés inhérentes à l'intérêt public dans la conservation (I), les enjeux de la conservation de la nature (II) enfin, l'étude envisagera quelques perspectives (III).

\section{Aperçu sur les aires protégées de la RDC}

Avant d'entrer dans le vif de ces aspects, force est de placer un mot sur l'historique de la conservation des aires protégées en RDC. L'histoire des aires protégées de la RDC est le fruit d'une longue période, s'étalant de sur plus d'un siecle et qui, par bien d'aspects est intimement liée à celle de l'Etat. ${ }^{28}$ En effet, à la fin du XIXème siècle et début XXème siècle, c'est fut la période caractérisée par l'émergence d'une reconnaissance internationale à propos de la nécessité de protéger certaines espèces d'une surexploitation. C'est ainsi que les premières réserves in situ ont vu le jour pour réglementer les prélèvements des bois, des quelques espèces des grands mammifères.

Le réseau actuel des aires protégées de la RDC comprend plus de 25 catégories nationales d'aires protégées in et ex situ. Les forêts du bassin du Congo constituent le deuxième plus grand massif de forêts tropicales au monde, après celui de l'Amazonie. Celles de la RDC représentent à elles seules environ $60 \%$ de ce massif forestier ${ }^{29}$. Comme l'ont montré les échanges lors de la Conférence Internationale sur la Gestion Durable des Forêts en RDC tenue à Bruxelles le 26 et 27 février 2007, ces forêts sont soumises à de nombreuses interventions tant pour leur conservation que pour leur exploitation artisanale et commerciale ${ }^{30}$. C'est le fait d'une multitude d'acteurs ayant chacun des objectifs particuliers et quelquefois opposés les uns aux autres. Outre ces aires protégées, le législateur a procédé à la protection des certaines ressources naturelles et biologiques et même aquatiques à des fins de protection et de conservation pour une utilisation rationnelle et durable de ces ressources. Cellesci sont contenues dans des dispositions éparses des instruments juridiques nationaux et internationaux auxquels en vigueur dont nous avions sus évoqués.

En effet, ladite loi dans sa partie relative aux mécanismes de protection, ne précise pas la manière dont l'Etat, les provinces et l'entité territoriale décentralisée doivent procéder à l'adoption des plans, stratégies de gestion et autres mesures nécessaires en vue de la restauration des écosystèmes dégradés et de favoriser la reconstitution des espèces menacées suivant les compétences qui sont les leurs. C'est dire que d'une part, toutes les personnes mo-

28 PELISSIER C., et al., Aires protégées de l'Afrique Centrale-état 2015, OFAC, Kinshasa, RDC et Yaoundé, Cameroun, 2016, p. 6.

29 DEVERS D., chercheur, University of Maryland, Observatoire Satellital des Forêts d'Afrique Centrale (OSFAC).

30 LEGRAND C. et BORRINI-FEYERABEND G., et al., Idéologie de l'intérêt général et Gouvernance des aires protégées : de la compréhension à l'action, UICN, Paris, 2015, p. 19. 
rales de droit public congolais sont superposées et d'autre part, bénéficient chacune d'une autonomie propre de gestion.

Au-delà de cette ambigüité, la province et l'entité territoriale décentralisée ne se retrouvent pas dans le processus dans l'octroi des dérogations gouvernementales dans le cas de l'intérêt public et pour des raisons de recherche scientifique où le gouvernement peut accorder des dérogations de permis et certificats prévus par les articles 64 à 67 de la loi cihaut évoquée. C'est ici l'occasion de rappeler que l'intérêt public auquel l'article 19 fait allusion rentre aussi dans ce que l'on appelle la compétence de la province ou de l'entité territoriale décentralisée. Cependant, autant qu'il existe un intérêt public national autant on peut parler de l'intérêt public provincial ou local.

L'intérêt public attaché à la protection de l'environnement procède d'une fonction de contrôle des actions étatiques. Reposant sur des mécanismes différents des fonctions d'encadrement. Il est toutefois intéressant de souligner que celles-ci contribuent toutes deux à l'effectivité du standard d'intérêt général et partant, de sa composante environnementale. La protection de l'environnement en tant que composante de l'intérêt général vient donc fonder la mise en place d'obligations positives à la charge de l'État. En effet, l'affaire Öneryildiz de 2002 illustre bien cette logique puisque qu'elle établit l'obligation positive de l'État de protéger la propriété d'un individu face à des nuisances environnementales.

\section{Difficultés de la conservation de la nature en RDC au regard du concept d'intérêt public}

Le défi majeur que fait face le droit de la conservation de la nature du fait de la notion d'intérêt public produit des obstacles liées à l'échelle de valeur qui existe entre d'une part, la nécessité de procéder à la prise des mesures de protection et de conservation de la nature pour l'intérêt public et d'autre part, les nécessités urgentes qui justifient les dérogations à ces mesures de conservation de la nature. L'emploi d'intérêt public qui est un concept mouvant et imprécis, à géométrie variable, pose problème au niveau de la compréhension du degré que le législateur s'accorde pour procéder à la prise des décisions et politiques publiques de la conservation de la nature.

De ces difficultés, la précaution doit être observée dans les actions, mesures et programmes de l'Etat et tous les autres acteurs publics et privés impliqués dans la gestion, la protection et la conservation de la nature en vue d'intégrer toutes les considérations actuelles de la conservation et de l'environnement.

\section{Enjeux et perspectives de la conservation de la nature en RDC au regard de la notion d'intérêt public}

Le droit de la conservation de la nature évolue en réponse à des besoins changeants de la société. La législation et les politiques publiques du pays à tous les niveaux de la prise de décision en matière de conservation de la nature continueront d'évoluer avec l'acquisition 
de nouvelles données scientifiques sur la biodiversité et sur l'impact des activités humaines, comme les modifications de l'utilisation des sols, ou de facteurs naturels, comme le changement climatique. Ce droit de la conservation de la nature répondra aussi à l'évolution du droit international, notamment avec l'adoption très récente des accords de Paris sur le changement climatique. Sur le plan administratif et judiciaire, des évolutions se produiront également, à mesure que l'Etat congolais, les acteurs environnementaux publics ou privés, les organisations environnementales nationales ou internationales les populations riveraines et les sociétés intègrent ces nouvelles connaissances dans la législation, programmes d'actions selon le cas.

\section{Conclusion}

La biosphère étant une entité qui implique une gestion globale, ne dépendant nullement des frontières politiques, c'est pourquoi il est de plus en plus pertinent d'entrevoir des solutions à tous les niveaux pour protéger et conserver efficacement la nature de l'échelle planétaire jusqu'à l'échelle locale en passant par le niveau régional et local. Le législateur congolais en procédant à l'adoption des lois sur la conservation de la nature pour des raisons d'intérêt public, reste compétent pour donner sens à la notion d'intérêt public et, doit être prudent au risque de créer une supercherie intellectuelle afin d'octroyer des prérogatives exorbitantes à l'Etat au détriment des autres entités politiques à savoir la province et l'ETD. Toutefois, l'avenir de la conservation de la nature passe par une précision claire de la notion d'intérêt public pour garantir une gouvernance efficace et durable des aires protégées, ressources naturelles, biologiques ainsi qu'aquatiques.

La bonne conservation de la nature en RDC implique une prise en compte globale et interdisciplinaire afin d'aboutir à une gestion durable et efficace des espèces de faune et de flore du pays. Pour ce faire, l'autorité compétente en matière de conservation de la nature a beaucoup plus besoin de l'implication de tous les acteurs publics ou privés, nationaux ou internationaux intervenants dans le secteur de la coservation de la nature. Il se dégage de la loi sur la conservation de la nature et d'autres lois et codes sus évoqués, que la notion d'intérêt public qui est une notion flou et à contenu variable est une supercherie intellectuelle que les autorités étatiques utilisent souvent abusivement pour justifier les mesures conservatoires et dérogatoires.

Au regard de la complexité de la notion d'intérêt public dans la gestion, la protection et la conservation de la nature en RDC, la bonne conservation des espèces de faune et de flore de la RDC nécessite une clarification de la part du législateur de cette notion d'intérêt public pour permettre aux autorités de l'Etat à tous les niveaux de prendre des mesures efficaces en vue de la conservation. En permettant aussi à ces entités de mener leurs activités dans le respect des textes légaux qui les régissent en lieu et place de favoriser certaines de ces entités (Pouvoir central) au détriment des autres (comme la province ou l'ETD). 